\title{
Feasibility of Conductivity Imaging Using Subject Eddy Currents Induced by Switching of MRI Gradients
}

\author{
Omer Faruk Oran and Yusuf Ziya Ider*
}

Purpose: To investigate the feasibility of low-frequency conductivity imaging based on measuring the magnetic field due to subject eddy currents induced by switching of MRI zgradients.

Methods: We developed a simulation model for calculating subject eddy currents and the magnetic fields they generate (subject eddy fields). The inverse problem of obtaining conductivity distribution from subject eddy fields was formulated as a convection-reaction partial differential equation. For measuring subject eddy fields, a modified spin-echo pulse sequence was used to determine the contribution of subject eddy fields to MR phase images.

Results: In the simulations, successful conductivity reconstructions were obtained by solving the derived convectionreaction equation, suggesting that the proposed reconstruction algorithm performs well under ideal conditions. However, the level of the calculated phase due to the subject eddy field in a representative object indicates that this phase is below the noise level and cannot be measured with an uncertainty sufficiently low for accurate conductivity reconstruction. Furthermore, some artifacts other than random noise were observed in the measured phases, which are discussed in relation to the effects of system imperfections during readout.

Conclusion: Low-frequency conductivity imaging does not seem feasible using basic pulse sequences such as spin-echo on a clinical MRI scanner. Magn Reson Med 77:1926-1937, 2017. (C) 2016 International Society for Magnetic Resonance in Medicine

Key words: eddy currents; gradient; conductivity; low frequency; image distortions; MRI

\section{INTRODUCTION}

Electrical conductivity varies among tissues and with frequency $(1,2)$. At low frequencies, the lipid membrane of cells acts as an insulator and prevents currents from entering cells, whereas at high frequencies, currents can pass through the capacitance of the cell membrane (3). This implies that the lower and upper frequency spectra of conductivity convey different information about tissues. High-frequency conductivity maps can be used to obtain local specific absorption rate maps (4), whereas low frequency conductivity maps can be used to monitor thermal therapeutic procedures (5), electroencephalogram source localization $(6,7)$, and the planning of trans-

Department of Electrical and Electronics Engineering, Bilkent University, Ankara, Turkey.

*Correspondence to: Yusuf Ziya Ider, Ph.D., Department of Electrical and Electronics Engineering, Bilkent University, Cankaya, 06800 Ankara, Turkey. E-mail: ider@ee.bilkent.edu.tr

Received 25 February 2016; revised 28 April 2016; accepted 28 April 2016 DOI 10.1002/mrm.26283

Published online 1 July 2016 in Wiley Online Library (wileyonlinelibrary.com).

(C) 2016 International Society for Magnetic Resonance in Medicine 1926 cranial magnetic stimulation (8-10). Furthermore, both high- and low-frequency conductivities depend on the pathological state of tissues; for example, conductivity maps may be used for the detection and characterization of tumors (11-18).

Several MRI-based techniques have been proposed for conductivity imaging at high and low frequencies. For high-frequency conductivity imaging, MR electrical properties tomography (MREPT) techniques constitute the largest class. In these techniques, the inverse problem of reconstructing the electrical properties (conductivity and permittivity) from the measured radiofrequency (RF) field $\left(B_{1}\right)$ is solved by exploiting the fact that $B_{1}$ field is perturbed by the underlying electrical properties of imaged subjects $(4,19-24)$. For low-frequency conductivity imaging, techniques classified as MR electrical impedance tomography (MREIT) are the most widely known (25-29). In these techniques, currents are injected into imaged subjects via surface electrodes. Magnetic fields generated by internal currents are measured, and this information is used for reconstructing conductivity.

In MREIT, current injection causes problems such as pain sensation and geometric distortions, which are triggered by denser current density near electrodes (27). To deal with these challenges, the induced-current MREIT technique has been proposed, in which electrical currents are induced inside imaged subjects by means of external coils (30). However, the use of external coils inside an MRI scanner limits the practicality of this technique. As a remedy for this problem, it has been proposed to use readily available MRI gradient coils for inducing "subject eddy currents" inside subjects (31-39). Subject eddy currents generate secondary magnetic fields, which are referred to as "subject eddy fields." Similar to MREIT, the ultimate purpose is to reconstruct conductivity from the measured subject eddy fields. However, no experimental conductivity reconstruction has been presented yet (31-39).

In this study, we investigated the feasibility of lowfrequency conductivity imaging using subject eddy currents induced by switching of the slice-selection gradient. The feasibility was investigated within the context of two main goals. The first goal was to understand whether conductivity reconstruction is possible, provided that subject eddy fields are measured accurately. To attain this goal, the inverse problem of obtaining the conductivity distribution from subject eddy fields was formulated as a convection-reaction partial differential equation (PDE). Successful conductivity reconstructions were obtained by solving this equation using simulated data. The second goal was to understand the fidelity by which subject eddy fields must be measured for 
accurately reconstructing conductivity. For measuring subject eddy fields, a modified spin-echo pulse sequence was proposed by which the contribution of subject eddy fields to MR phase images is determined. We found that this contribution cannot be measured with an uncertainty sufficiently low for accurate conductivity reconstruction. In addition to the random noise, some biased artifacts were observed in the phase measurements. These artifacts were modeled by considering the effects of undesired magnetic fields due to system imperfections during readout.

\section{THEORY}

\section{Definition and Properties of System and Subject Eddy Currents}

Due to switching of gradients, "system eddy currents" are induced on the metallic system components of an MRI scanner such as RF and gradient shields, coils, thermal shields of the magnet, and the magnet itself (40). System eddy currents generate "system eddy fields" $\left(\mathbf{B}_{\text {sys }}\right)$ and the decay of $\mathbf{B}_{\text {sys }}$ after a gradient ramp can be modeled by using exponential functions with different amplitudes and time constants ranging from a few milliseconds to a few seconds (40). By means of the gradient waveform pre-emphasis and actively shielded gradient coils, $\mathbf{B}_{\text {sys }}$ is significantly lowered within the imaging volume (41).

In addition to system eddy currents, the electric fields (E), which are induced due to switching of gradient fields $\left(\mathbf{B}_{p}\right)$, give rise to subject eddy currents $\left(\mathbf{J}_{\text {sub }}\right)$ in imaged subjects which in turn generate subject eddy fields ( $\left.\mathbf{B}_{\text {sub }}\right)$. The governing equations for $\mathbf{J}_{\text {sub }}$ and $\mathbf{B}_{\text {sub }}$ are obtained using three assumptions: 1) The contribution of $\mathbf{B}_{\text {sys }}$ to $\mathbf{E}$ is negligible inside imaged subjects, because $\mathbf{B}_{\text {sys }}$ is $0.05 \%$ (or less) of $\mathbf{B}_{p}$ in the imaging volume (41). 2) $\mathbf{B}_{\text {sub }}$ is significantly lower than $\mathbf{B}_{p}$, and thus its contribution to $\mathbf{E}$ is also negligible (this assumption is validated by the levels of simulated $\mathbf{B}_{\text {sub }}$ ). 3) Displacement currents are negligible compared with conductive currents because of the low-frequency nature of $\mathbf{B}_{p}$. Therefore, inside the imaged subject, the governing equations during switching of $\mathbf{B}_{p}$ are obtained as

$$
\begin{gathered}
\nabla \times \mathbf{E}(\mathrm{t}) \cong-\frac{\partial \mathbf{B}_{\mathrm{p}}(\mathrm{t})}{\partial t} \\
\nabla \times \mathbf{B}_{\text {sub }}(\mathrm{t}) \cong \mu_{0} \sigma \mathbf{E}(\mathrm{t})=\mu_{0} \mathbf{J}_{\text {sub }}(\mathrm{t})
\end{gathered}
$$

where $\sigma$ is the conductivity distribution of the imaged subject, and the magnetic permeability of the imaged subject is taken as $\mu_{0}$. Because of the assumption that $\mathbf{B}_{\text {sub }} \ll \mathbf{B}_{\mathrm{p}}$, Equations 1.1 and 1.2 become uncoupled, and thus subject eddy currents can be assumed to be instantly vanishing after the gradient ramp, which is in contrast to slowly decaying system eddy currents (39). Although the contribution of $\mathbf{B}_{\text {sys }}$ to $\mathbf{E}$ is negligible, $\mathbf{B}_{\text {sys }}$ itself can cause significant phase accumulation in the MRI phase images $(40,41)$.
Measurement of Subject Eddy Fields Due to Slice-Selection Gradients

A modified spin-echo pulse sequence is used for measuring the phase accumulated by subject eddy fields due to switching of slice-selection gradients (see Fig. 1). The sliceselection direction is taken as the $z$-direction. Because the z-gradient is linearly ramped up or down with the same slew rate at all edges, the subject eddy field is constant, and its magnitude is equal at all edges, as is evident from Equations 1.1 and 1.2. Considering the net contributions only (Fig. 1), the accumulated phase $\left(\varphi_{\text {sub,z }}\right)$ is obtained as

$$
\varphi_{\text {sub }, \mathrm{z}}=\gamma B_{\text {sub }, \mathrm{z}}\left(\tau_{\mathrm{exc}}+2 \tau_{\mathrm{rfc}}\right)
$$

where $\gamma$ is the gyromagnetic ratio, $B_{\text {sub,z }}$ is the $z$-component of the subject eddy field due to switching of the z-gradient, and $\tau_{\text {exc }}$ and $\tau_{\mathrm{rfc}}$ are the relevant ramp times shown in Figure $1\left(B_{\text {sub,z }}\right.$ will be hereafter referred to as the "subject eddy field"). When $G_{z}^{+}$and $G_{z}^{-}$are used in two separate measurements (see Fig. 1), the acquired MR phase images can be expressed as

$$
\begin{aligned}
\varphi^{+}(x, y)= & \varphi_{\text {sub }, \mathrm{z}}(x, y)+\varphi_{\mathrm{RF}}(x, y)+\varphi_{\text {sys }, \mathrm{z}}(x, y) \\
& +\varphi_{\text {other }}(x, y) \\
\varphi^{-}(x, y)= & -\varphi_{\text {sub }, \mathrm{z}}(x, y)+\varphi_{\mathrm{RF}}(x, y)-\varphi_{\text {sys }, \mathrm{z}}(x, y) \\
& +\varphi_{\text {other }}(x, y)
\end{aligned}
$$

where $\varphi_{\mathrm{RF}}$ is the phase of the RF field (transceive phase of the $B_{1}$ field), $\varphi_{\text {sys }, z}$ is the phase accumulated by the $z$ component of the system eddy field due to switching of the $z$-gradient $\left(B_{\mathrm{sys}, \mathrm{z}}\right)$, and $\varphi_{\mathrm{other}}$ is the phase accumulated by the sum of system and subject eddy fields due to switching of other gradients (because a spin-echo pulse sequence is used, the main magnetic field $\left[\mathrm{B}_{0}\right]$ inhomogeneity does not have a net contribution to the accumulated phase). If the measurements using $G_{z}^{+}$and $G_{z}^{-}$are also performed for a nonconductive phantom in which $\varphi_{\text {sub,z }}$ is zero, $\varphi_{\text {sub,z }}$ can be obtained as

$$
\begin{gathered}
\left\{\frac{\varphi^{+}(x, y)-\varphi^{-}(x, y)}{2}\right\}_{\sigma \neq 0}=\varphi_{\text {sub }, \mathrm{z}}+\varphi_{\text {sys }, \mathrm{z}} \\
\left\{\frac{\varphi^{+}(x, y)-\varphi^{-}(x, y)}{2}\right\}_{\sigma=0}=\varphi_{\text {sys }, \mathrm{z}} \\
\left.\left\{\frac{\varphi^{+}(x, y)-\varphi^{-}(x, y)}{2}\right\}_{\sigma \neq 0}\right\}_{\sigma=0}=\varphi_{\text {sub }, \mathrm{z}}
\end{gathered}
$$

where $\sigma$ denotes the conductivity. Once $\varphi_{\text {sub,z }}$ is measured, Equation 2 may be used with known values of $\tau_{\text {exc }}$ and $\tau_{\text {rfc }}$ for obtaining $B_{\text {sub }, z}$.

\section{Modeling Effects of System Imperfections During Readout}

The $z$-component of the system eddy field due to switching of the $z$-gradient $\left(B_{\mathrm{sys}, z}\right)$ and the $z$-component of other undesired magnetic fields $\left(B_{\text {other,z }}\right)$, including system 


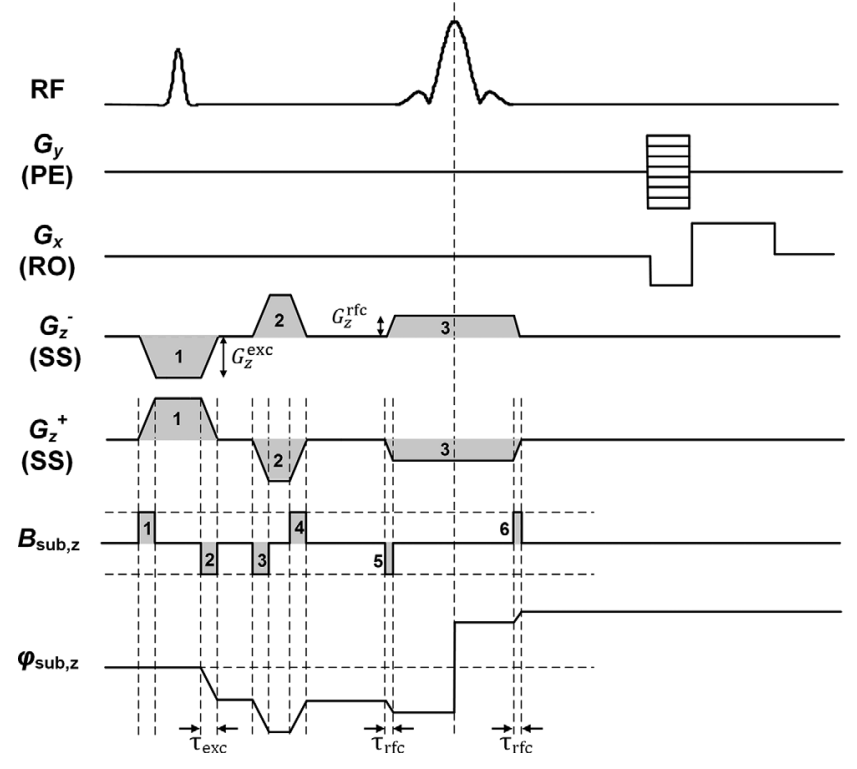

FIG. 1. The "modified" spin-echo pulse sequence for measuring the phase accumulated by the $z$-component of the subject eddy field $\left(B_{\text {sub }, z}\right)$, which is induced due to switching of the $z$-gradient. The ramping times of the $z$-gradient field are exaggerated for a better visualization of $B_{\text {sub } z}$. For increasing the accumulated phase $\left(\varphi_{\text {sub }, z}\right)$, the third lobe of the $z$-gradient is applied in the opposite direction of the first lobe, which is in contrast to a conventional spin-echo sequence (this is why the proposed sequence is called "modified"). Two separate measurements, one using $G_{z}^{+}$ and one using $G_{z}^{-}$, are performed. The waveforms of $B_{\text {sub.z }}$ and $\varphi_{\text {sub }, z}$ for the case of $G_{z}^{+}$are shown at the sixth and seventh rows. The first lobe of $B_{\text {sub.z }}$ does not contribute to $\varphi_{\text {sub.z }}$ since it is before the excitation and the contribution of the third and fourth lobes cancel each other. On the other hand, the fifth and sixth lobes, which are opposite each other, both contribute to $\varphi_{\text {sub.z }}$ because of the refocusing RF pulse applied in between. Consequently, only the second, fifth, and sixth lobes of $B_{\text {sub.z }}$ have a net contribution to $\varphi_{\text {sub,z }}$, and this contribution is determined by the ramp times $\tau_{\text {exc }}$ and $\tau_{\text {rfc }}$. The values of $G_{z}^{\text {exc }}, G_{z}^{\text {rfc }}, \tau_{\text {exc }}$, and $\tau_{\text {rfc }}$ are provided in Table 1. PE, phase encoding; RF, radiofrequency field; $\mathrm{RO}$, readout; SS, slice selection.

eddy fields due to switching of $x$ - and $y$-gradients and the $\mathrm{B}_{0}$ inhomogeneity, manifest themselves with geometric distortions such as shifting, scaling, or shearing in the reconstructed image, because $B_{\text {sys,z }}$ and $B_{\text {other,z }}$ also exist during readout $(41,42)$. It can be safely assumed that $B_{\text {sys }, z}$ and $B_{\text {other,z }}$ are constant in time during the short readout window ( $\sim 1-2 \mathrm{~ms})$. Including the effects of geometric distortions, the phase images in Equations 3.1 and 3.2 become (42)

$$
\begin{aligned}
& \varphi^{+}\left(x^{+}, y\right)=\varphi^{+}\left(x+\frac{B_{\text {other }, z}(x, y)}{G_{x}}+\frac{B_{\text {sys }, z}(x, y)}{G_{x}}, y\right) \\
& \varphi^{-}\left(x^{-}, y\right)=\varphi^{-}\left(x+\frac{B_{\text {other }, z}(x, y)}{G_{x}}-\frac{B_{\text {sys }, z}(x, y)}{G_{x}}, y\right)
\end{aligned}
$$

where $\varphi^{+}\left(x^{+}, y\right)$ and $\varphi^{-}\left(x^{-}, y\right)$ are the phase images with geometric distortions, the readout direction is assumed to be the $x$-direction, and $G_{x}$ is the readout gradient field. Because $\varphi^{+}\left(x^{+}, y\right)$ and $\varphi^{-}\left(x^{-}, y\right)$ are not aligned due to $B_{\text {sys }, z}(x, y) / G_{x}$ term, their difference contains biased artifacts. For cylindrical phantoms with uniform conductivity, these artifacts can be modeled by considering the terms given in Equations 3.1 and 3.2. For this purpose, similar to the approach employed by Mandija et al. (38), $\varphi_{\mathrm{RF}}$ is approximated with a quadratic function (43) [i.e., $\varphi_{\mathrm{RF}}(x, y)=b_{1}^{\sigma=\sigma_{0}}+b_{2}^{\sigma=\sigma_{0}}\left(x^{2}+y^{2}\right)$ where $\sigma_{0}$ is the conductivity of the phantom (the coefficients scale with the conductivity of the phantom (43)]. Substituting $x^{+}$ or $x^{-}$for $x$ in $\varphi_{R F}(x, y)$, the difference between $\varphi^{+}\left(x^{+}, y\right)$ and $\varphi^{-}\left(x^{-}, y\right)$ are expressed as

$$
\begin{aligned}
& \left\{\frac{\varphi^{+}\left(x^{+}, y\right)-\varphi^{-}\left(x^{-}, y\right)}{2}\right\}_{\sigma=\sigma_{0}} \\
& =\frac{\varphi_{\text {sub }, \mathrm{z}}^{\text {sum }}}{2}+\frac{\varphi_{\text {sys }, \mathrm{z}}^{\text {sum }}}{2}+\frac{\varphi_{\text {ofther }}^{\text {diff }}}{2}+2 b_{2}^{\sigma=\sigma_{0}}\left(x+\frac{B_{\text {other }, \mathrm{z}}}{G_{X}}\right) \frac{B_{\text {sys }, \mathrm{z}}}{G_{X}}
\end{aligned}
$$

where $\varphi_{\text {sub }, \mathrm{z}}^{\text {sum }}=\varphi_{\text {sub }, \mathrm{z}}\left(x^{+}, y\right)+\varphi_{\text {sub }, \mathrm{z}}\left(x^{-}, y\right), \varphi_{\text {sys }, \mathrm{z}}^{\text {sum }}=\varphi_{\text {sys }, \mathrm{z}}\left(x^{+}\right.$, $y)+\varphi_{\text {sys. }}\left(x^{-}, y\right)$ and $\varphi_{\text {other }}^{\text {diff }}=\varphi_{\text {other }}\left(x^{+}, y\right)-\varphi_{\text {other }}\left(x^{-}, y\right)$. Because of the misregistration between $\varphi^{+}\left(x^{+}, y\right)$ and $\varphi^{-}\left(x^{-}, y\right)$, additional terms appear in Equation 6 compared with the terms in Equation 4.1.

As discussed in the previous section, $\varphi^{+}$and $\varphi^{-}$ should also be measured for a nonconductive phantom. However, preparing a phantom material with zero conductivity may not be practical. Therefore, we assume that a low-conductive phantom is used instead of a nonconductive phantom. Considering Equation 6 for the low-conductive phantom, $\varphi_{\text {sys, }}^{\text {sum }}$ and $\varphi_{\text {other }}^{\text {diff }}$ are the same as in the conductive phantom, whereas $\varphi_{\text {sub.z }}^{\text {sum }}$ and the last term in Equation 6 are different because they depend on the conductivity. Assuming that $\varphi_{\text {sub, }, \mathrm{sum}} / 2 \cong \varphi_{\text {sub }, \mathrm{z}}$ (see the Appendix for the validation), the measured phase $\left(\varphi_{\text {meas }}\right)$, which would ideally equal $\varphi_{\text {sub,z }}$, can be expressed as (compare with Equation 4.3)

$$
\begin{aligned}
& \varphi_{\text {meas }}=\left\{\frac{\varphi^{+}\left(x^{+}, y\right)-\varphi^{-}\left(x^{-}, y\right)}{2}\right\}_{\sigma=\sigma_{0}} \\
& -\left\{\frac{\varphi^{+}\left(x^{+}, y\right)-\varphi^{-}\left(x^{-}, y\right)}{2}\right\}_{\sigma=\sigma_{\text {low }}} \\
& \cong \varphi_{\text {sub }, z}^{\sigma_{0}-\sigma_{\text {low }}}+2\left(b_{2}^{\sigma=\sigma_{0}}-b_{2}^{\sigma=\sigma_{\text {low }}}\right)\left(x+\frac{B_{\text {other }, z}}{G_{X}}\right) \frac{B_{\text {sys }, z}}{G_{x}}
\end{aligned}
$$

where $\sigma_{\text {low }}$ is the conductivity of the low-conductive phantom and $\quad \varphi_{\text {sub, },}^{\sigma_{0}-\sigma_{\text {low }}}=\left\{\varphi_{\text {sub }, \mathrm{z}}\right\}_{\sigma=\sigma_{0}}-\left\{\varphi_{\text {sub, },}\right\}_{\sigma=\sigma_{\text {low }}}$. Because the second term in Equation 7 is related to the $\mathrm{RF}$ phase that could not be eliminated, it is referred to as the "RF leakage" as it was in the study by Mandija et al. (38). Note that the RF leakage scales with $\left(b_{2}^{\sigma=\sigma_{0}}-b_{2}^{\sigma=\sigma_{\text {low }}}\right.$ ) and thereby also scales with the phantom conductivity difference $\left(\sigma_{0}-\sigma_{\text {low }}\right)(43)$.

\section{Conductivity Reconstruction from Subject Eddy Fields}

A novel method for conductivity reconstruction is developed that is based on the solution of the following central equation (for the derivation, see the Appendix):

$$
\nabla \rho \cdot\left(-J_{\text {sub }, y}, J_{\text {sub }, \mathrm{x}}\right)+\rho \frac{\nabla^{2} B_{\text {sub }, \mathrm{z}}}{\mu_{0}}=\frac{\partial B_{\mathrm{p}, \mathrm{z}}(\mathrm{t})}{\partial t}
$$


where $\rho$ is the resistivity $\left(\rho=\sigma^{-1}\right), J_{\text {sub,x }}$ and $J_{\text {sub,y }}$ are the $x^{-}$and $y$-components of the subject eddy current, and $B_{\text {sub,z }}$ and $B_{\mathrm{p}, \mathrm{z}}$ are the $\mathrm{z}$-components of the subject eddy field and the gradient field, respectively. Equation 8 is in the form of a convection-reaction equation and is similar to the equation that has been derived previously for the case of using switching of readout gradients (32). For solving Equation 8, $J_{\text {sub,x }}$ and $J_{\text {sub,y }}$ must be reconstructed beforehand. They are related to $B_{\text {sub.z }}$ with the following relation (for the derivation, see the Appendix):

$$
\frac{\nabla^{2} B_{\text {sub }, \mathrm{z}}}{\mu_{0}}=\frac{\partial J_{\text {sub,x }}}{\partial y}-\frac{\partial J_{\text {sub }, \mathrm{y}}}{\partial x}
$$

Equation 9 is the same as the fundamental relation used in the MR current density imaging (MRCDI) (44), and thus any MRCDI algorithm may be used. In this study, the MRCDI algorithm proposed by Park et al. was used (45), in which the following PDE is solved for $\beta$ at the imaging slice (the inner region of the slice is denoted by $\Omega$, and its boundary is denoted by $\partial \Omega$ ).

$$
\begin{aligned}
& \nabla^{2} \beta=\frac{\nabla^{2} B_{\text {sub,z }}}{\mu_{0}} \text { in } \Omega \\
& \beta=0 \text { on } \partial \Omega
\end{aligned}
$$

The $x$ - and $y$-components of the reconstructed subject eddy current $\left(J_{\text {sub,x }}^{*}\right.$ and $J_{\text {sub,y }}^{*}$ ) are obtained from $\left(J_{\text {sub }, \mathrm{x}}^{*}, J_{\text {sub }, y}^{*}\right)=\left(\frac{\partial \beta}{\partial y},-\frac{\partial \beta}{\partial x}\right)$. Because $J_{\text {sub,z }}$ and some components of $J_{\text {sub,x }}$ and $J_{\text {sub,y }}$ do not generate $B_{\text {sub,z }}$, they are undetectable. Therefore, $\mathbf{J}^{*}=\left(J_{\text {sub }, \mathrm{x}}^{*}, J_{\text {sub }, \mathrm{y}}^{*}, 0\right)$ is only an estimate to $\mathbf{J}_{\text {sub }}$, and the overall error in the reconstructed subject eddy current is proportional to $\left\|J_{\text {sub,z }}\right\|$ and $\left\|\frac{\partial J_{\text {sub, } z}}{\partial z}\right\|$ at the imaging slice (45).

On the other hand, in regions where conductivity slowly varies, $\nabla \rho$ in Equation 8 can be neglected as done in Wen's MREPT formula (46), and conductivity can be directly reconstructed using the following pointwise formula:

$$
\sigma \cong \frac{\nabla^{2} B_{\mathrm{sub}, \mathrm{z}}}{\mu_{0} \partial B_{\mathrm{p}, \mathrm{z}}(\mathrm{t}) / \partial t}
$$

\section{Analysis of Uncertainty in the Reconstructed Conductivity}

In this analysis, for the sake of simplicity, it is assumed that the conductivity is slowly varying within a region of interest, and Equation 11 is modified as (for details, see the Appendix)

$$
\sigma \cong \frac{\nabla^{2} \varphi_{\text {sub }, z}}{\gamma \mu_{0} z_{0}\left(G_{z}^{\mathrm{exc}}+2 G_{z}^{\mathrm{rfc}}\right)}
$$

where $\varphi_{\text {sub,z }}$ is the phase due to subject eddy fields, $G_{z}^{\text {exc }}$ and $G_{z}^{\mathrm{rc}}$ are as defined in Table 1 , and $z_{0}$ is the $z$-coordinate of the imaging slice. Our goal was to identify a relationship between the uncertainties of $\sigma$ and $\varphi_{\text {sub.z }}$, which are denoted by $\mathrm{u}(\sigma)$ and $\mathrm{u}\left(\varphi_{\text {sub,z }}\right)$. It is assumed that $\nabla^{2}$ $\varphi_{\text {sub,z }}$ is calculated through the convolution of $\varphi_{\text {sub,z }}$ with a $5 \times 5 \times 3$ Savitzky-Golay Laplacian kernel. Therefore, the $\nabla^{2} \varphi_{\text {sub,z }}$ value in one pixel is the linear combination of the
Table 1

Experimental Parameters.

\begin{tabular}{lc}
\hline Parameter & Setting \\
\hline Field of view, mm & $256 \times 256(224 \times 224)$ \\
Matrix size & $128 \times 128(32 \times 32)$ \\
Voxel size, mm & $2 \times 2 \times 5(7 \times 7 \times 5)$ \\
Imaging slice, transverse & $z=0.13 \mathrm{~m}$ \\
Echo time, ms & 10 \\
Repetition time, ms & 1000 \\
Flip angle & $90^{\circ}$ \\
Number of acquisitions & 16 \\
Total imaging time, min & $34.1 \times 4(8.5 \times 4)$ \\
Bandwidth, Hz/pixel & 500 \\
$G_{x}$ readout, $\mathrm{mT} / \mathrm{m}$ & $5.9(1.7)$ \\
$\mathrm{G}_{z}^{\text {exc }}$ excitation, $\mathrm{mT} / \mathrm{m}$ & $15.66^{\mathrm{a}}$ \\
$\mathrm{G}_{z}^{\text {rc }}$ refocusing, $\mathrm{mT} / \mathrm{m}$ & $4.8^{\mathrm{a}}$ \\
Slew rate of the $z-g r a d i e n t, ~ T / m / s$ & $160^{\mathrm{b}}$ \\
Excitation ramp time $\tau_{\text {exc }}, \mu \mathrm{s}$ & 98 \\
Refocusing ramp time $\tau_{\mathrm{rfc}}, \mu \mathrm{s}$ & 30
\end{tabular}

For the settings which were different in the first and second sets of experiments, the parenthetical settings are for the second set. ${ }^{a}$ Given that the slice thickness and the flip angle are kept the same, if the z-gradient is increased, the bandwidth of the RF pulses should be increased by applying narrower RF pulses in time, which requires higher output voltage of the RF amplifier. For the MRI scanner used in this study, the $z$-gradient values of 15.66 $\mathrm{mT} / \mathrm{m}$ and $4.8 \mathrm{mT} / \mathrm{m}$ were constrained by the output voltage of the $\mathrm{RF}$ amplifier rather than the maximum allowed $z$-gradient.

${ }^{\mathrm{b}}$ The slew rate is the same in every edge of the $z$-gradient field. Note that if the slew rate increases, the instantaneous subject eddy field increases while the ramp times decrease. Therefore, the phase accumulated due to the subject eddy field remains the same, because it is found by the time integral of subject eddy field (see Eq. 2). In other words, the accumulated phase during one ramp is determined by the end-value of the gradient field rather than its slew rate.

$\varphi_{\text {sub,z }}$ values within the neighborhood of that pixel, which is defined by the size of the kernel. Assuming that the noise distributions for each pixel of $\varphi_{\text {sub,z }}$ are independent and identically distributed (47), and using the law of error propagation (48), it is found that

$$
\mathrm{u}(\sigma)=\frac{\mathrm{u}\left(\varphi_{\text {sub,z }}\right)}{\gamma \mu_{0} z_{0}\left(G_{z}^{e x c}+2 G_{z}^{r f c}\right)}\left[\frac{2}{105 \Delta x^{4}}+\frac{2}{105 \Delta y^{4}}+\frac{6}{25 \Delta z^{4}}\right]^{1 / 2}
$$

where $\Delta x$ and $\Delta y$ are the voxel sizes in the $x$ - and $y$ directions, and $\Delta z$ is the slice thickness. Note that the Laplacian kernel amplifies the high-frequency noise components, and $\mathrm{u}\left(\varphi_{\text {sub,z }}\right)$ is thereby increased by a factor of $\left[\frac{2}{105 \Delta x^{4}}+\frac{2}{105 \Delta y^{4}}+\frac{6}{25 \Delta z^{4}}\right]^{1 / 2}$. This factor is obtained from the analytically calculated elements of the Savitzky-Golay kernel (48). It has been shown that, when the Savitzky-Golay Laplacian kernel is used, this factor becomes minimum compared with any other Laplacian kernel of the same size (48).

\section{METHODS}

Numerical Methods for Simulations and Conductivity Reconstruction

For calculation of subject eddy currents and fields, the 'Magnetic Fields' module of the finite element method 


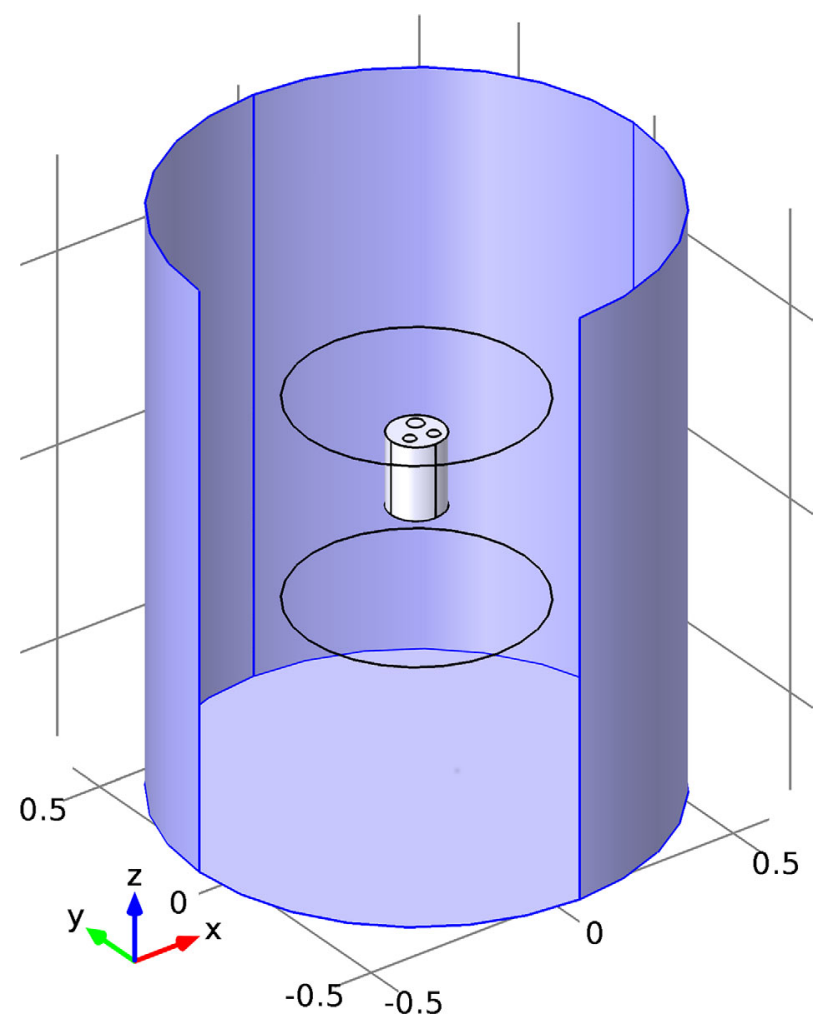

FIG. 2. Illustration of the COMSOL Multiphysics model used for calculating subject eddy currents and subject eddy fields. The outermost cylinder, which has a radius of $60 \mathrm{~cm}$ and a height of $300 \mathrm{~cm}$, is the solution domain on which the tangential component of the vector magnetic potential is taken as zero. The $z$-gradient coil is obtained with the wire model of a Maxwell pair. The simulation phantom, which has a radius of $7 \mathrm{~cm}$ and a height of $19 \mathrm{~cm}$, is also shown. The phantom is placed along the $z$-direction and its base is located at $z=-0.02 \mathrm{~m}$ plane. The imaging slice is chosen as the $z=0.13 \mathrm{~m}$ plane, where the maximum subject eddy field occurs among other transversal slices. The background conductivity of the phantom is taken as $0.5 \mathrm{~S} / \mathrm{m}$ and three cylindrical regions of conductivity anomaly are assumed along the phantom (see Fig. 3a for the assigned conductivities of these regions).

package COMSOL Multiphysics 4.2a (COMSOL AB, Stockholm, Sweden) was used (system eddy fields were not simulated in this study). In this module, in contrast to Equation 1.1, it is not assumed that subject eddy fields $\left(\mathbf{B}_{\text {sub }}\right)$ are negligible compared with gradient fields $\left(\mathbf{B}_{p}\right)$. Instead, the following time-dependent PDE was solved for the total vector magnetic potential (A) in a large domain that contained the $z$-gradient coil and the simulation phantom (Fig. 2). Note that $\mathbf{A}$ is defined as $\mathbf{A}=\mathbf{A}_{p}+\mathbf{A}_{\text {sub }}$, where $\nabla \times \mathbf{A}_{p}=\mathbf{B}_{p}$ and $\nabla \times \mathbf{A}_{\text {sub }}=\mathbf{B}_{\text {sub }}$.

$$
\nabla \times \nabla \times \mathbf{A}(\mathrm{t})=\mathbf{J}_{\text {coil }}(t)-\sigma(x, y) \frac{\partial \mathbf{A}(\mathrm{t})}{\partial t}
$$

In this equation, $\sigma(x, y)$ is the conductivity distribution, which is zero in regions other than the simulation phantom, and $\mathbf{J}_{\text {coil }}$ is the current density, which is running on the z-gradient coil and which generates the gradient field. In solving Equation 14, the temporal gauge was used, in which the scalar electric potential vanishes (49). Therefore, the subject eddy current was obtained from the calculated A using $\mathbf{J}_{\text {sub }}(\mathrm{t})=-\sigma(x, y) \frac{\partial \mathbf{A}}{\partial t}$. In order to obtain $\mathbf{B}_{\text {sub }}$, Equation 14 was solved again with zero phantom conductivity; therefore, $\nabla \times \mathbf{A}=\mathbf{B}_{p}$, because $\mathbf{B}_{\text {sub }}=0$. This $\mathbf{B}_{p}$ field was then subtracted from the previously calculated $\nabla \times \mathbf{A}$, which equals $\mathbf{B}_{p}+\mathbf{B}_{\text {sub }}$.

We used a cylindrical simulation phantom that had a radius of $7 \mathrm{~cm}$, a height of $19 \mathrm{~cm}$, and three cylindrical conductivity anomalies (Fig. 2). The z-gradient coil was modeled as a Maxwell pair [the radius of each of the circular coils was $30 \mathrm{~cm}$, and they were separated by $30 \sqrt{3}$ $\mathrm{cm}$ (50)]. The waveform of $\mathbf{J}_{\text {coil }}$ was assigned such that the $z$-gradient field linearly ramped up with a slew rate of $160 \mathrm{~T} / \mathrm{m} / \mathrm{s}$, and thus $\partial \mathbf{B}_{\mathrm{p}}(\mathrm{t}) / \partial t$ was constant. In the simulations performed using a step size of $1 \mathrm{~ns}$, it was observed that $\mathbf{B}_{\text {sub }}$ ramped up or down in 10 ns (i.e., almost instantly) (39) and stayed constant between ramps because of the constant $\partial \mathbf{B}_{\mathrm{p}}(\mathrm{t}) / \partial t$. Because we were interested in $\mathbf{B}_{\text {sub }}$ during its plateau, a larger step size of $0.1 \mu \mathrm{s}$ was used, and a few time-steps were sufficient for reaching the plateau.

The computed $z$-component of the subject eddy field $\left(B_{\text {sub.z }}\right)$ was exported to MATLAB (MathWorks, Natick, Massachusetts, USA) from COMSOL Multiphysics. Equations 8 and 10 were solved using the finite difference method, which was implemented in MATLAB (24). The Laplacian of $B_{\text {sub.z }}$ was calculated using a threedimensional $(5 \times 5 \times 3)$ Savitzky-Golay Laplacian kernel (51). In solving Equation 8, the Dirichlet boundary condition was used, and the $\rho$ values of the boundary were assigned known resistivity of the background. Because there was no diffusion term in Equation 8, its numerical solution suffers from unwanted oscillations near interior and boundary layers (52). As a remedy for this problem, an artificial diffusion term $\left(-\mathrm{c} \nabla^{2} \rho\right)$ was added to Equation 8 , which is a well-known stabilization technique (52). The c coefficient of $5 \times 10^{-5}$ was chosen such that the oscillations vanished, yet no significant smoothing was introduced in the reconstructed conductivity $(24,28)$.

\section{Experimental Methods}

Three homogeneous cylindrical phantoms, each with a radius of $7 \mathrm{~cm}$ and a height of $19 \mathrm{~cm}$, were constructed using agar-saline gels containing $18 \mathrm{~g} / \mathrm{L}$ agar, $1.5 \mathrm{~g} / \mathrm{L}$ $\mathrm{CuSO}_{4}$, and different concentrations of $\mathrm{NaCl}$ (6, 9, and $0 \mathrm{~g} / \mathrm{L}$ for the first, second, and third phantoms, respectively). The agar-saline gels were solid enough to neglect flow artifacts. The conductivity of the three phantoms were measured as $1.3,1.6$, and $0.2 \mathrm{~S} / \mathrm{m}$ using the phasebased MREPT technique proposed by Voigt et al. (19). This technique estimates the conductivity at the Larmour frequency (123.2 MHz for our scanner). However, it has been reported that the conductivity of agar-saline gels do not change significantly in the range of $10-200 \mathrm{MHz}$ (53-55). Therefore, these estimates were also representative at low frequencies. Although the third phantom was intended to be nonconductive, we obtained a conductivity of $0.2 \mathrm{~S} / \mathrm{m}$ due to agar.

Two sets of experiments were performed using the proposed pulse sequence (Fig. 1), which was implemented on a 3T MRI scanner (Magnetom Trio, Siemens Healthcare, Erlangen, Germany). For transmit and 

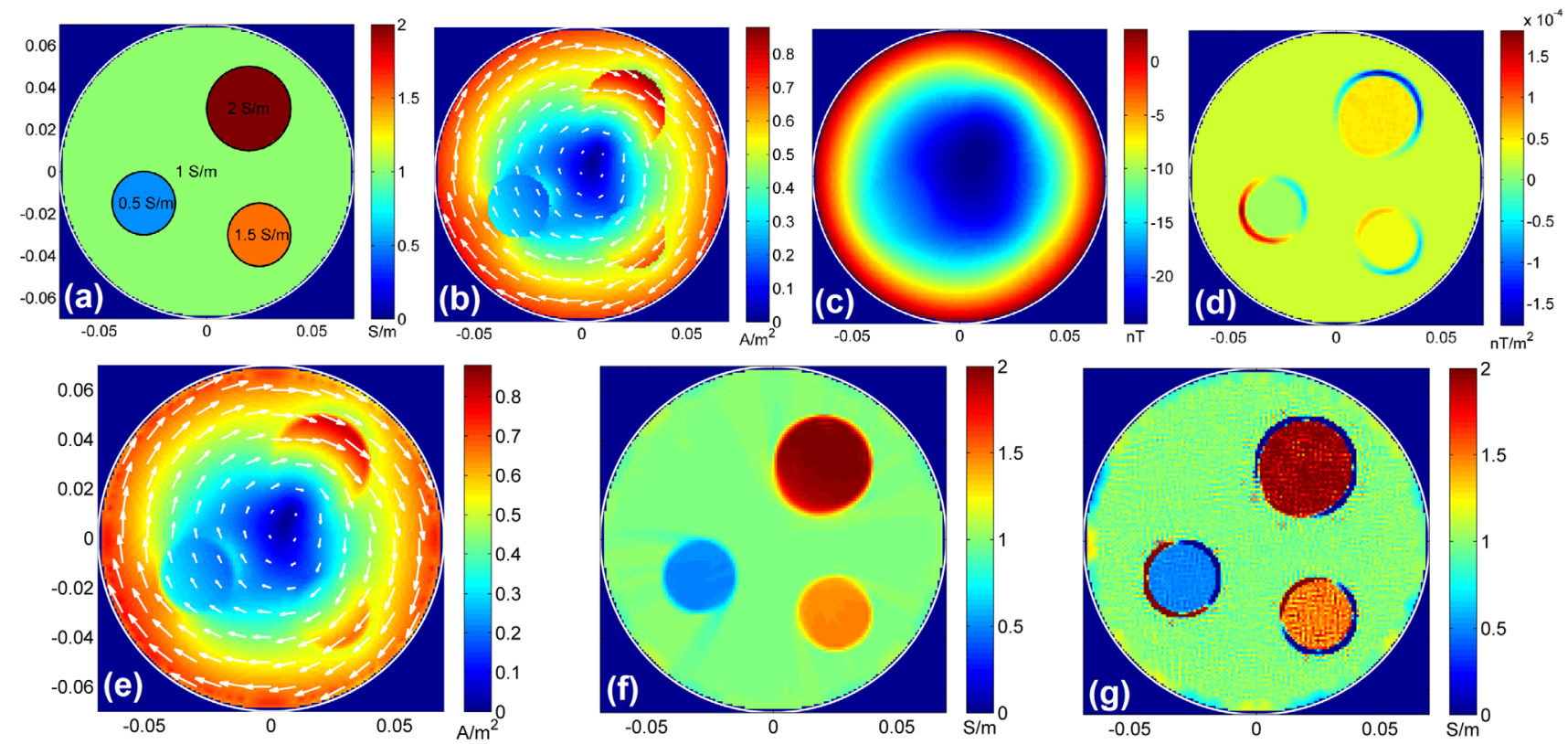

FIG. 3. Simulation results at the imaging slice $(z=0.13 \mathrm{~m}$ plane) of the cylindrical phantom which has three conductivity anomaly regions. (a) Actual conductivity distribution. (b) Magnitude distribution and vector plot of the actual subject eddy current. (c) Distribution of the $z$-component of the subject eddy field $\left(B_{\text {sub.z }}\right)$. (d) Distribution of the Laplacian of $B_{\text {sub.z. }}$. (e) Magnitude distribution and vector plot of the subject eddy current, which is reconstructed by solving Equation 10. (f) Distribution of the conductivity, which is reconstructed by solving the convectionreaction equation (Eq. 8). (g) Distribution of the conductivity distribution, which is reconstructed by the pointwise formula (Eq. 11).

receive, the quadrature birdcage body coil of the MRI scanner was used. The phantoms were placed along the $z$-direction (the direction of $B_{0}$ was assumed as the $z$ direction). The slice orientation was transverse and the reference (center) slice was $2 \mathrm{~cm}$ away from the base of the phantom. The imaging slice was chosen as the $z=0.13 \mathrm{~m}$ plane; the other imaging parameters are summarized in Table 1. By constructing a platform on the patient table of the scanner, it was assured that the phantoms were fixed at the same position in all measurements, rendering artifacts due to phantom misalignments negligible.

The first set of experiments was performed in order to determine the uncertainty in the measured phase and to understand whether this uncertainty was sufficiently low for accurate conductivity reconstruction. In this set, the voxel size was $2 \times 2 \times 5 \mathrm{~mm}$. Because $\varphi_{\text {meas }}$ is the linear combination of four MRI phase images (see Eq. 7), its uncertainty $\left[\mathrm{u}\left(\varphi_{\text {meas }}\right)\right]$ can be obtained by noting the fact that the uncertainty of an MRI phase image equals the inverse of the signal-to-noise ratio (SNR) measured from the magnitude image of the same measurement (47). We have

$$
\mathrm{u}\left(\varphi_{\text {meas }}\right)=\frac{\sqrt{2}}{2} \sqrt{\frac{1}{\mathrm{SNR}_{\sigma=\sigma_{0}}{ }^{2}}+\frac{1}{\mathrm{SNR}_{\sigma=0.2}^{2}}}
$$

where $\mathrm{SNR}_{\sigma=\sigma_{0}}$ denotes the SNR of the magnitude image for either the first or second phantom ( $\sigma_{0}$ is the conductivity of the phantom) and $\mathrm{SNR}_{\sigma=0.2}$ denotes the SNR of the magnitude image for the third phantom. These SNR values were measured using the "SNR mult" method described by Dietrich et al. (56). Note that $\varphi_{\text {meas }}$ contained the RF leakage in addition to $\varphi_{\text {sub,z }}$ (see Eq. 7).
However, as is evident from Equation 15, $\mathrm{u}\left(\varphi_{\text {meas }}\right)$ is not affected by RF leakage [i.e., we would have seen the same $\mathrm{u}\left(\varphi_{\text {meas }}\right)$ if no $\mathrm{RF}$ leakage had existed in $\left.\varphi_{\text {meas }}\right]$. Therefore, for a desired uncertainty in the reconstructed conductivity, $\mathrm{u}(\sigma)_{\text {des }}$, the maximum allowed uncertainty in the measured phase, $\mathrm{u}\left(\varphi_{\text {meas }}\right)_{\max }$, can be obtained by rearranging Equation 13 as

$$
\begin{aligned}
& \mathrm{u}\left(\varphi_{\text {meas }}\right)_{\max }=\mathrm{u}(\sigma)_{\text {des }} \gamma \mu_{0} z_{0}\left(G_{z}^{e x c}+2 G_{z}^{r f C}\right) \\
& {\left[\frac{2}{105 \Delta x^{4}}+\frac{2}{105 \Delta y^{4}}+\frac{6}{25 \Delta z^{4}}\right]^{-1 / 2}}
\end{aligned}
$$

The second set of experiments were performed for investigating $\mathrm{RF}$ leakage. In this set, large voxels $(7 \times 7 \times 5 \mathrm{~mm})$ were used for assuring low $\mathrm{u}\left(\varphi_{\text {meas }}\right)$ so that the RF leakage was not obscured by noise in $\varphi_{\text {meas }}$. Specifically, using Equation 15 and the measured SNR values (56)—which are 1960, 1421, and 2940 for the first, second and third phantoms- $-\mathrm{u}\left(\varphi_{\text {meas }}\right)$ was estimated as $4.3 \times 10^{-4} \mathrm{rad}$ and $5.5 \times 10^{-4} \mathrm{rad}$ for the first and second phantoms, respectively. These uncertainties were sufficiently lower than the level of measured RF leakages as will be elaborated in "Experimental Results" section.

The RF leakage in the measured phase scaled with the $\left(b_{2}^{\sigma=\sigma_{0}}-b_{2}^{\sigma=0.2}\right)$ coefficient as shown by Equation 7. This coefficient was measured by fitting a quadratic function to the difference $\left\{\varphi^{+}(x, y)\right\}_{\sigma=\sigma_{0}}-\left\{\varphi^{+}(x, y)\right\}_{\sigma=0.2}$ (see Eq. 3.1). Because the same $z$-gradient polarity was used, there was no misregistration between the phase images, and the terms other than $\varphi_{\mathrm{RF}}$ canceled each other in the difference $\left(\varphi_{\text {sub,z }}\right.$ is negligible compared with $\left.\varphi_{R F}\right)$. Therefore, this difference equaled $b_{0}^{\sigma=\sigma_{0}}-b_{0}^{\sigma=0.2}+\left(b_{2}^{\sigma=\sigma_{0}}-b_{2}^{\sigma=0.2}\right)\left(x^{2}+y^{2}\right)$. The fitted $\left(b_{2}^{\sigma=1.3}\right.$ 

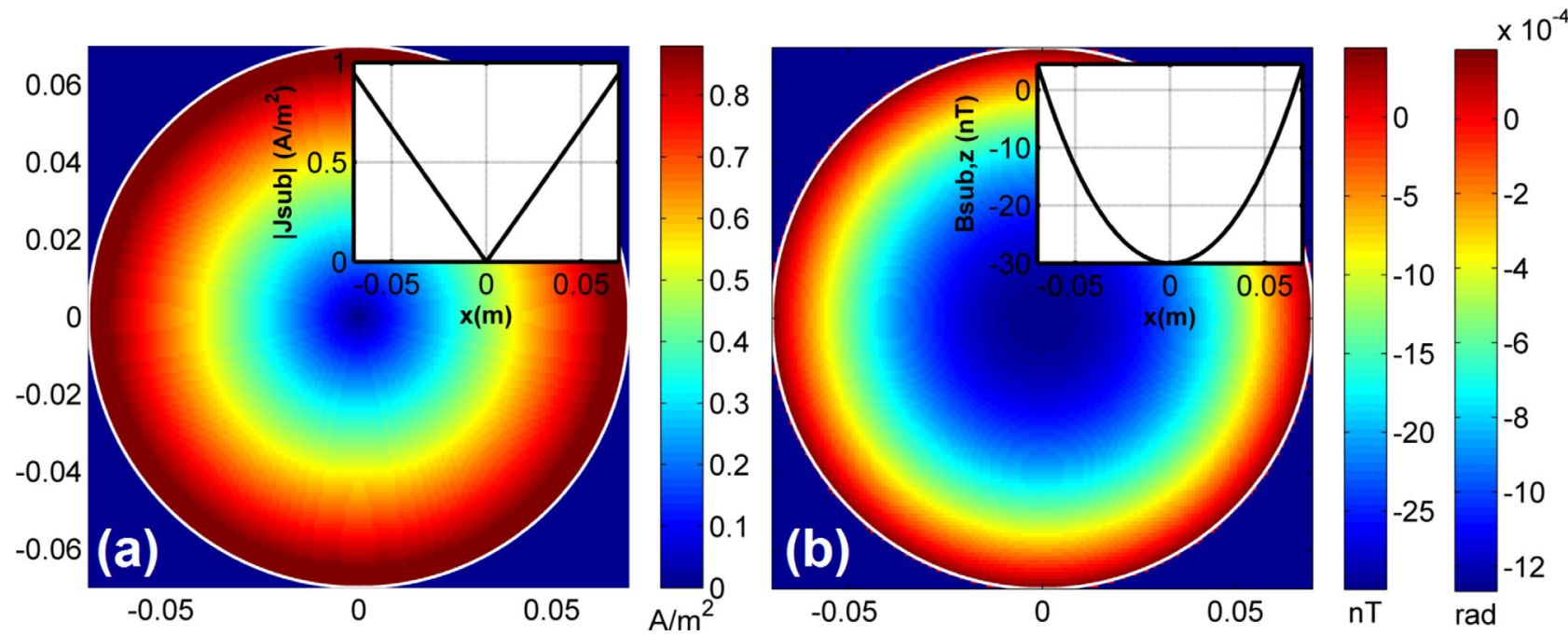

FIG. 4. Simulation results for the first experimental phantom $(\sigma=1.3 \mathrm{~S} / \mathrm{m})$ at the imaging slice $(z=0.13 \mathrm{~m}$ plane $)$ when a linear gradient ramp with a slew rate of $160 \mathrm{~T} / \mathrm{m} / \mathrm{s}$ was assumed. (a) Distribution and profile of the subject eddy current magnitude. The profile is plotted along the $y=0$ line. (b) Distribution and profile of the $z$-component of the subject eddy field $\left(B_{\text {sub.z. }}\right)$. The profile is plotted along the $y=0$ line. The phase accumulated due to $B_{\text {sub }, z}$ is calculated using Equation 2 with the ramp times $\left(\tau_{\text {exc }}\right.$ and $\left.\tau_{\text {rfc }}\right)$ given in Table 1 . The scale of this phase distribution is represented by the rightmost color bar.

$-b_{2}^{\sigma=0.2}$ ) coefficient was $271 \mathrm{rad} / \mathrm{m}^{2}$, whereas the fitted $\left(b_{2}^{\sigma=1.6}-b_{2}^{\sigma=0.2}\right)$ coefficient was $339 \mathrm{rad} / \mathrm{m}^{2}$.

\section{RESULTS}

\section{Simulation Results}

Figure 3 shows the results obtained using the simulation phantom, which had three cylindrical anomaly regions. Figure $3 b-3 d$ shows the distributions of the subject eddy current magnitude $\left(\left|\mathbf{J}_{\text {sub }}\right|\right)$, the subject eddy field $\left(B_{\text {sub,z }}\right)$, and $\nabla^{2} B_{\text {sub,z }}$ at the imaging slice $(z=0.13$ plane) when the slew rate of the gradient field was $160 \mathrm{~T} / \mathrm{m} / \mathrm{s}$. As expected from Equation $8, \nabla^{2} B_{\text {sub,z }}$ attains high magnitudes at the anomaly boundaries where conductivity changes abruptly. Because a $5 \times 5 \times 3$ Savitzky-Golay kernel was used in the calculation of $\nabla^{2} B_{\text {sub,z }}$, the highmagnitude $\nabla^{2} B_{\text {sub,z }}$ regions at the boundaries are 5 pixels wide.

Figure 3e and 3f shows the distribution of the reconstructed subject eddy current magnitude $\left(\left|\mathbf{J}_{\text {sub }}^{*}\right|\right)$ and the reconstructed conductivity, which were obtained by solving Equations 10 and 8. The relative $L^{2}$ errors in the reconstructed $\left|\mathbf{J}_{\text {sub }}^{*}\right|$ and in the reconstructed conductivity were $3.1 \%$ and $6.1 \%$, respectively. The reconstruction errors were most pronounced in the regions near the anomaly boundaries. This is due to the fact that the actual conductivities jumped at these boundaries, whereas the reconstructed ones changed more smoothly. The smoothing was caused by the Savitzky-Golay kernel and by the artificial diffusion term of Equation 8, both of which have low-pass filter effects (57). The relative $L^{2}$ errors in the reconstructed conductivity and $\left|\mathbf{J}_{\text {sub }}^{*}\right|$ were less than $1 \%$ if the errors were calculated in a region where the actual conductivity was constant. Figure $3 f$ shows the reconstructed conductivity when the pointwise formula given in Equation 11 is used, in which the spatial variation of the conductivity is neglected. As expected, artifacts were observed at the boundaries of the anomalies. This result suggests that the contribution of the convective term in Equation 8 is significant, especially at the internal boundaries.

Simulations were also performed for the experimental phantoms. The position and geometry of the gradient coil were the same as in the previous case, and the phantom was assumed at the same position. Figure $4 \mathrm{a}$ and $4 \mathrm{~b}$ shows the distributions of the subject eddy current magnitude and the subject eddy field at the imaging slice $(z=0.13 \mathrm{~m}$ plane) for the first experimental phantom $(\sigma=1.3 \mathrm{~S} / \mathrm{m})$ when a linear gradient ramp with a slew rate of $160 \mathrm{~T} / \mathrm{m} / \mathrm{s}$ was assumed. The subject eddy field $\left(B_{\text {sub.z }}\right)$, which was constant in time during the gradient ramp, had a maximum magnitude of $30 \mathrm{nT}$. When the proposed pulse sequence was assumed to be used (Fig. 1), $\varphi_{\text {sub,z }}$ accumulated by this $B_{\text {sub,z }}$ was calculated by using Equation 2 with the ramp times $\left(\tau_{\text {exc }}\right.$ and $\left.\tau_{\mathrm{rfc}}\right)$ given in Table 1. It was assumed that the slew rate of the $z$-gradient was $160 \mathrm{~T} / \mathrm{m} / \mathrm{s}$ at all edges of the $z$-gradient. The maximum magnitudes of the expected $\varphi_{\text {sub,z }}$ were $1.3 \times 10^{-3} \operatorname{rad}\left(0.075^{\circ}\right)$ and $1.6 \times 10^{-3} \operatorname{rad}\left(0.092^{\circ}\right)$ for the first and second phantoms, respectively.

\section{Experimental Results}

Using the proposed pulse sequence (Fig. 1) and the experimental phantoms, two sets of experiments were performed. In the first set, a voxel size of $2 \times 2 \times 5 \mathrm{~mm}$ was used. The SNR of the MR magnitude images (when either of the $G_{z}^{+}$and $G_{z}^{-}$is used) were calculated as 160 , 116 and 240 for the first, second, and third phantoms respectively. Using these SNR values in Equation 15, $\mathrm{u}\left(\varphi_{\text {meas }}\right)$ was estimated as $5.3 \times 10^{-3}$ and $6.1 \times 10^{-3} \mathrm{rad}$ for the first and second phantoms, respectively. These uncertainties are nearly four times the maximum magnitudes of the expected $\varphi_{\text {sub,z }}$, and thus $\varphi_{\text {sub,z }}$ is below the noise level. Furthermore, for achieving an uncertainty of 

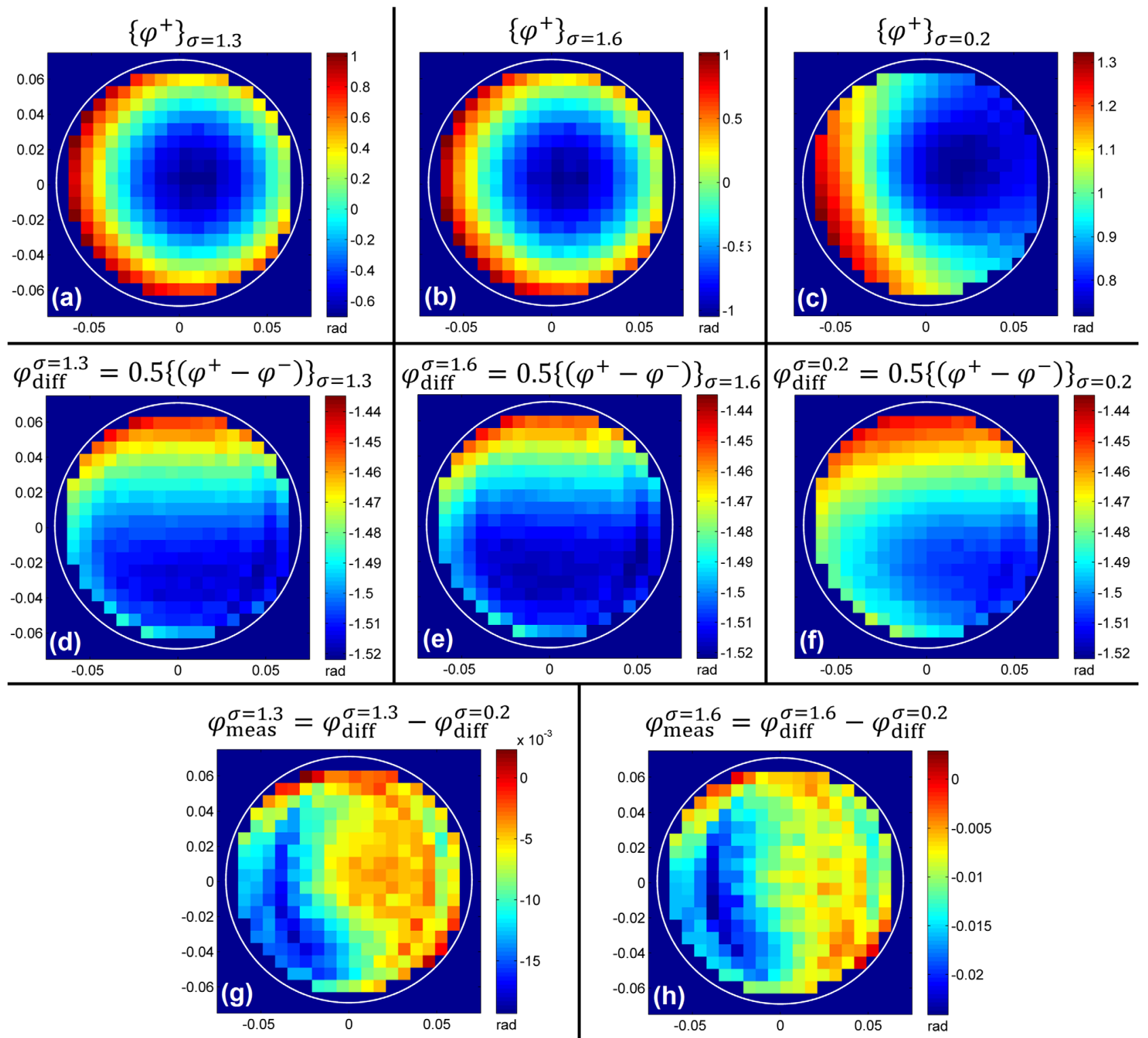

FIG. 5. Experimental results regarding the demonstration of the RF leakage at the imaging slice $(z=0.13 \mathrm{~m}$ plane $)$ of the experimental phantoms The units of the $x$-axis and $y$-axis are meters. (a-c) MR phase images of the first $(\sigma=1.3 \mathrm{~S} / \mathrm{m})(\mathrm{a}), \mathrm{second}(\sigma=1.6 \mathrm{~S} / \mathrm{m})(\mathrm{b})$, and third $(\sigma=0.2 \mathrm{~S} / \mathrm{m})$ (c) phantoms when the polarity of the $z$-gradient was positive (see $G_{z}^{+}$in Fig. 1). (d-f) Half of the difference between the phase images acquired using positive and negative $z$-gradient polarities for the three phantoms $\left(\varphi_{\text {diff }}^{\sigma=1.3}, \varphi_{\text {diff }}^{\sigma=1.6}\right.$, and $\left.\varphi_{\text {diff }}^{\sigma=0.2}\right)$. (g) Measured phase for the first phantom $\left(\varphi_{\text {meas }}^{\sigma=1.3}=\varphi_{\text {diff }}^{\sigma=1.3}-\varphi_{\text {diff }}^{\sigma=0.2}\right)$. (h) Measured phase for the second phantom $\left(\varphi_{\text {meas }}^{\sigma=1.6}=\varphi_{\text {diff }}^{\sigma=1.6}-\varphi_{\text {diff }}^{\sigma=0.2}\right)$.

$0.1 \mathrm{~S} / \mathrm{m}$ in the reconstructed conductivity, the maximum allowed uncertainty of $\varphi_{\text {meas }}$, which is denoted by $\mathrm{u}\left(\varphi_{\text {meas }}\right)_{\max }$, is calculated as $2.1 \times 10^{-7}$ rad using Equation 16 with the experimental parameters given in Table $1(0.1 \mathrm{~S} / \mathrm{m}$ is assumed to be an acceptable uncertainty for the reconstructed conductivity). Because the $\mathrm{u}\left(\varphi_{\text {meas }}\right)$ values of the two phantoms are significantly larger than $\mathrm{u}\left(\varphi_{\text {meas }}\right)_{\max }$, the conductivity reconstruction using data from the experiments is not feasible. Even when large voxel sizes are used (such as $7 \times 7 \times 5 \mathrm{~mm}$ ), the achievable $\mathrm{u}\left(\varphi_{\text {meas }}\right)$ is still three orders of magnitude more than $\mathrm{u}\left(\varphi_{\text {meas }}\right)_{\max }$.

The second set of experiments were performed to demonstrate biased artifacts in $\varphi_{\text {meas }}$, which are thought to be associated with RF leakage. Figure $5 a-5 c$ shows the MR phase images at the imaging slice $(z=0.13 \mathrm{~m})$ of the three phantoms when $G_{z}^{+}$was used (the phase images for $G_{z}^{-}$are not shown). For the three phantoms, Figure $5 \mathrm{~d}-5 \mathrm{f}$ shows half the difference between the MRI phase images acquired using $G_{z}^{+}$and $G_{z}^{-}$. These difference images, which are labeled as $\varphi_{\text {diff }}^{\sigma=1.3}, \varphi_{\text {diff }}^{\sigma=1.6}$, and $\varphi_{\text {diff }}^{\sigma=0.2}$, may be modeled by using Equation 6. Among the terms of Equation $6, \varphi_{\text {sub, }}^{\text {sum }}$ was not observable with the scale of these figures because it was in the order of $10^{-3}$ rad (see Fig. $4 \mathrm{~b}$ ), and the $\varphi_{\text {sys,z }}^{\text {sum }}$ and $\varphi_{\text {other }}^{\text {diff }}$ terms were the same for all the phantoms because they did not depend on conductivity. Therefore, if only the first three terms of Equation 6 were observed, we would have obtained the same 
distributions for $\varphi_{\text {diff }}^{\sigma=1.3}, \varphi_{\text {diff }}^{\sigma=1.6}$, and $\varphi_{\text {diff }}^{\sigma=0.2}$, but that was not the case. It may be hypothesized that these differences are accounted for in the last term in Equation 6, which is the RF leakage term. Figure $5 \mathrm{~g}$ shows the difference between $\varphi_{\text {diff }}^{\sigma=1.3}$ and $\varphi_{\text {diff }}^{\sigma=0.2}$, whereas Figure $5 \mathrm{~h}$ shows the difference between $\varphi_{\text {diff }}^{\sigma=1.6}$ and $\varphi_{\text {diff }}^{\sigma=0.2}$. These differences, which were defined as $\varphi_{\text {meas }}$, were modeled using Equation 7. The levels of $\varphi_{\text {meas }}$ were in the order of $10^{-2}$ rad, indicating that the contribution of $\varphi_{\text {sub,z }}^{\sigma_{0}-\sigma_{\text {low }}}$ was not dominant (see Fig. 4b). Therefore, we expected $\varphi_{\text {meas }}$ to be proportional to the coefficients $\left(b_{2}^{\sigma=1.3}-b_{2}^{\sigma=0.2}\right)$ and $\left(b_{2}^{\sigma=1.6}-b_{2}^{\sigma=0.2}\right)$. As expected, the pattern of $\varphi_{\text {meas }}$ was similar for two phantoms, and the ratio between their levels was approximately 0.8 , which equals the experimentally calculated ratio of $\frac{\left(b_{2}^{\sigma=1.3}-b_{2}^{\sigma=0.2}\right)}{\left(b^{\sigma=1.6}-b^{\sigma=0.2}\right)}=\frac{271}{339}=0.8$. Therefore, we may argue that the theory of RF leakage expressed through Equations 6 and 7 is consistent with our observations.

\section{DISCUSSION}

This feasibility study had two goals. The first goal was to investigate whether conductivity reconstruction is possible provided that subject eddy fields $\left(B_{\text {sub,z }}\right)$ are measured accurately. For this purpose, a novel conductivity reconstruction method was developed by formulating a convection-reaction type PDE which relates conductivity to $B_{\text {sub,z }}$. The simulation results show that the proposed method performs well both in regions of homogeneous conductivity and in regions of high conductivity gradients (see Fig. 3). The second goal was to understand the fidelity by which $\varphi_{\text {sub,z }}$ must be measured in order to accurately reconstruct conductivity. It was observed that the uncertainty of the measured phases $\left[\mathrm{u}\left(\varphi_{\text {meas }}\right)\right]$ were nearly four times the maximum magnitudes of expected $\varphi_{\text {sub,z }}$. Furthermore, $\mathrm{u}\left(\varphi_{\text {meas }}\right)$ was four orders of magnitude higher than the maximum allowed $\mathrm{u}\left(\varphi_{\text {meas }}\right)$ for an accurate conductivity reconstruction. Indeed, in human experiments, $\mathrm{u}\left(\varphi_{\text {meas }}\right)$ will not be so different, because the expected SNR is on the same order of magnitude as that of the phantoms. This leads us to the key conclusion of this study, which is that the low-frequency conductivity imaging using slice-selection gradients is not feasible, at least not for the experimental procedures we applied.

As a by-product of this study, biased artifacts observed in $\varphi_{\text {meas }}$ were also investigated by developing a theoretical model that hypothesizes that these artifacts are caused by the RF phase, which could not be eliminated due to misregistration between $\varphi^{+}\left(x^{+}, y\right)$ and $\varphi^{-}\left(x^{-}, y\right)$. The developed model suggests that these artifacts, which are referred to as RF leakage, scale with conductivity of phantoms. Indeed, such scaling is also observed in our experiments, which supports the validity of the model. Therefore, even if $\mathrm{u}\left(\varphi_{\text {meas }}\right)$ may be sufficiently lowered by some innovative means, there are still nonrandom RF leakage artifacts that must be handled in order to accurately measure $\varphi_{\text {sub,z }}$ and reconstruct conductivity.

The level of RF leakage depends on the extent of geometric distortions, because this extent is determined by $B_{\text {sys, } \mathrm{z}} / G_{X}$ and $B_{\text {other }, \mathrm{z}} / G_{X}$ (see Equations $5.1,5.2$, and 7 ). Consider, for instance, the RF leakage for the first phantom, which is on the level of $20 \times 10^{-3} \mathrm{rad}$ (see Fig. 5g). If
$B_{\text {other,z }} / G_{X}$ is neglected and if $B_{\text {sys,z }} / G_{X}$ is assumed to be spatially constant, the RF leakage can be expressed as $2 x\left(b_{2}^{\sigma=1.3}-b_{2}^{\sigma=0.2}\right) s_{0}$, where $s_{0}$ is the shift caused by constant $B_{\text {sys,z }} / G_{x}$. The RF leakage level of $20 \times 10^{-3}$ rad can be reached with $s_{0} \cong 0.5 \mathrm{~mm}$ when $x$ changes between -7 and $7 \mathrm{~cm}$ (the radius of the phantom is $7 \mathrm{~cm}$ ). Therefore, even small geometric distortions can generate RF leakages high enough to dominate $\varphi_{\text {sub,z }}$. Furthermore, the pattern of the RF leakage indicates that these small geometric distortions are not in the form of a simple shift, but that higher-order distortions exist, which are harder to detect. Indeed, comparing the MRI magnitude images obtained using positive and negative $z$-gradient polarities (voxel size, $2 \times 2 \times 5 \mathrm{~mm}$ ), we have not observed nor detected any difference pointing to geometric distortions. Because the distortions are undetectable, we have not attempted any of the geometric distortion correction methods proposed in the literature $(41,42,58,59)$.

Measurement of subject eddy fields due to switching of gradients has been investigated in other studies as well (31-39). Mandija et al. (38) studied the measurement of the subject eddy field due to switching of readout $(x-$ or $y$-) gradients. They proposed measuring the phase accumulated due to this subject eddy field by taking the difference of two phase images acquired using spin-echo pulse sequences of opposite readout gradient polarities $\left(\varphi^{+}\right.$and $\left.\varphi^{-}\right)$. In another recent study, Gibbs and Liu (39) investigated the phase accumulated due to the subject eddy field induced by the falling edge of a gradient pulse that is applied immediately before the excitation. In the both studies, the level of accumulated phases is found to be below the noise level $(38,39)$, which is in line with our findings. Moreover, by applying subvoxel shifts to $\varphi^{+}$and $\varphi^{-}$via spatial interpolation, Mandija et al. also showed that even minor subvoxel misregistrations in $\varphi^{+}$and $\varphi^{-}$can lead to RF leakages that dominate desired measurements and scale with conductivity (38). Our experimental findings of RF leakage are also in line with the findings of this study.

It is known that the conductivities of certain biological tissues such as muscle or white matter are anisotropic at low frequencies $(2,60)$. For the reconstruction of anisotropic conductivity, some methods have been developed in the field of MREIT (61-63). In these methods, currents are injected through different pairs of surface electrodes so that several independent (not collinear) current density distributions are generated inside the subject in separate experiments, and the magnetic fields due to each current density distribution are measured. In the algorithm proposed by Nam et al. (62), the measured magnetic fields are first used to reconstruct the corresponding current density distributions. Starting from an initial estimate, the anisotropic conductivity tensor is then updated iteratively so that the solution of the forward problem matches these reconstructed current densities (the forward problem is defined as the calculation of current density for a given conductivity tensor). This methodology can potentially be adapted to the case of subject eddy current-based conductivity imaging. For this purpose, subject eddy currents induced by different gradient coils may be used as independent current density distributions. The subject eddy field due to the $z^{-}$ 
gradient coil can first be measured using the method described in the current study. Then, the subject eddy fields due to the $x$ - and $y$-gradient coils can be measured separately using the method described in other studies $(31,38)$. Using these subject eddy fields, three independent subject eddy current distributions can be reconstructed by the method based on the solution of Equation 10. Finally, the anisotropic conductivity tensor can be reconstructed iteratively by making use of the forward problem formulation in Equation 14. This method may be considered as one way of approaching anisotropic conductivity reconstruction in future eddy current-based conductivity reconstruction studies.

In conclusion, we have shown by way of simulation that the conductivity reconstruction using $B_{\mathrm{sub}, \mathrm{z}}$ is possible, provided that $\varphi_{\text {sub,z }}$ is measured accurately. However, we have also shown that $\varphi_{\text {sub,z }}$ cannot be measured with an uncertainty sufficiently low for accurate conductivity reconstruction, which indicates that the conductivity imaging using switching of slice-selection gradients is not feasible. We have come across nonrandom artifacts in $\varphi_{\text {meas }}$, which are hypothesized to be caused by RF leakage. We were able to show through experiments that RF leakage scales with conductivity as suggested by the developed model. On the other hand, the pattern of RF leakage is difficult to predict, because it requires exact knowledge of $B_{0}$ inhomogeneity and system eddy fields during readout. The complete verification of the RF leakage hypothesis, therefore, requires further study.

\section{APPENDIX}

\section{Derivation of Equations 8, 9, and 12}

For deriving Equation 9, the curl of both sides of Equation 1.2 is taken. Using the identity $\nabla \times \nabla \times \mathbf{B}_{\text {sub }}=\nabla(\nabla$ $\left.\cdot \mathbf{B}_{\text {sub }}\right)-\nabla^{2} \mathbf{B}_{\text {sub }}$ and noting that $\nabla \cdot \mathbf{B}_{\text {sub }}=0$, we obtain the following relation:

$$
-\nabla^{2} \mathbf{B}_{\text {sub }}=\mu_{0}\left(\nabla \times \mathbf{J}_{\text {sub }}\right)
$$

The z-component of Equation A.1 can be recognized as Equation 9. For deriving Equation 8, we use $\mathbf{J}_{\text {sub }}=\sigma \mathbf{E}$ in Equation A.1 to get

$$
-\nabla^{2} \mathbf{B}_{\text {sub }}=\mu_{0}(\nabla \sigma \times \mathbf{E}+\sigma \nabla \times \mathbf{E})
$$

Using Equation 1.1 and the relation $\mathbf{E}=\mathbf{J}_{\text {sub }} / \sigma$, the z-component of Equation A.2 becomes

$$
\nabla^{2} B_{\text {sub }, \mathrm{z}}=\frac{\mu_{0}}{\sigma}\left(\frac{\partial \sigma}{\partial y} J_{\text {sub }, \mathrm{x}}-\frac{\partial \sigma}{\partial x} J_{\text {sub }, \mathrm{y}}\right)+\mu_{0} \sigma \frac{\partial B_{\mathrm{p}, \mathrm{z}}(\mathrm{t})}{\partial t}
$$

Substituting $\rho=\sigma^{-1}$ with Equation A.3, Equation 8 is obtained by some algebraic manipulation.

For deriving Equation 12, we use Equation 2 in Equation 11 and note that, when the $z$-gradient is linearly ramped up or down with the same slew rate of $K$ at all edges, $\partial B_{\mathrm{p}, \mathrm{z}}(\mathrm{t}) / \partial t$ can be expressed at the imaging slice as $K z_{0}$, where $z_{0}$ is the $z$-coordinate of the imaging slice. In this case, Equation 11 can be expressed as

$$
\sigma \cong \frac{\nabla^{2} \varphi_{\text {sub }, \mathrm{z}}}{\mu_{0} \gamma\left(\tau_{\mathrm{exc}}+2 \tau_{\mathrm{rfc}}\right) K z_{0}}
$$

The $\left(\tau_{\text {exc }}+2 \tau_{\text {rfc }}\right) K$ term in Equation A.4 can be recognized as $G_{z}^{\text {exc }}+2 G_{z}^{\text {rc }}$, where $G_{z}^{\text {exc }}$ and $G_{z}^{\text {rfc }}$ are the magnitude of the $z$-gradient during excitation and refocusing, respectively (see Table 1). Substituting $G_{z}^{\mathrm{exc}}+2 G_{z}^{\mathrm{rfc}}$ for $\left(\tau_{\text {exc }}+2 \tau_{\text {rfc }}\right) K$ in Equation A.4, Equation 12 is obtained.

\section{Validation of the Assumption $\varphi_{\text {sub }, z}^{\text {sum }} / 2=\varphi_{\text {sub }, z}(x, y)$}

For cylindrical phantoms with uniform conductivity, $\varphi_{\text {sub,z }}$ can be approximated with a quadratic function (see the profile in Fig. 4b) [i.e., $\varphi_{\text {sub,z }}(x, y)=u_{1}^{\sigma=\sigma_{0}}+$ $\left.u_{2}^{\sigma=\sigma_{0}}\left(x^{2}+y^{2}\right)\right]$. Substituting $x^{+}$or $x^{-}$for $x, \varphi_{\text {sub, },}^{\text {sum }} / 2$ can be expressed as (for definition of $x^{+}$and $x^{-}$, see Equations 5.1 and 5.2)

$$
\begin{aligned}
\frac{\varphi_{\text {sub }, \mathrm{z}}^{\text {sum }}}{2}= & \varphi_{\text {sub }, \mathrm{z}}(x, y) \\
& +u_{2}^{\sigma=\sigma_{0}}\left[2 x \frac{B_{\text {other }, \mathrm{z}}}{G_{X}}+\left(\frac{B_{\text {other }, \mathrm{z}}}{G_{X}}\right)^{2}+\left(\frac{B_{\text {sys }, \mathrm{z}}}{G_{X}}\right)^{2}\right]
\end{aligned}
$$

Because $B_{\text {other. } \mathrm{Z}} / G_{X}$ and $B_{\mathrm{sys}, \mathrm{Z}} / G_{X}$ is on the order of 0.005 or less (41), $\left(B_{\text {other, }} / G_{X}\right)^{2}$ and $\left(B_{\text {sys }, Z} / G_{X}\right)^{2}$ can safely be neglected compared with $\left(x^{2}+y^{2}\right)$. Away from the origin, the $2 x B_{\text {other, } \mathrm{z}} / G_{x}$ term can also be neglected compared with $\left(x^{2}+y^{2}\right)$ (around the origin, the $u_{1}^{\sigma=\sigma_{0}}$ term is dominant in $\varphi_{\text {sub,z }}$ anyway). Therefore, the artifacts due to misregistration of $\varphi_{\text {sub,z }}$ are negligible compared with $\varphi_{\text {sub }, \mathrm{z}}$ itself, and thus $\varphi_{\text {sub }, \mathrm{z}}^{\text {sum }} / 2 \cong \varphi_{\text {sub }, \mathrm{z}}(x, y)$.

\section{ACKNOWLEDGMENTS}

Experimental data were acquired using the facilities of UMRAM (National Magnetic Resonance Research Center), Bilkent University, Ankara, Turkey.

\section{REFERENCES}

1. Gabriel C, Gabriel S, Corthout E. The dielectric properties of biological tissues: I. Literature survey. Phys Med Biol 1996;41:2231-2249.

2. Gabriel S, Lau RW, Gabriel C. The dielectric properties of biological tissues: II. Measurements in the frequency range $10 \mathrm{~Hz}$ to $20 \mathrm{GHz}$. Phys Med Biol 1996;41:2251-2269.

3. Pethig R. Dielectric properties of body tissues. Clin Phys Physiol Meas 1987;(8 Suppl A):5-12.

4. Katscher U, Voigt T, Findeklee C, Vernickel P, Nehrke K, Dossel O. Determination of Electric Conductivity and Local SAR Via B1 Mapping. IEEE Trans Med Imaging 2009;28:1365-1374.

5. Kwon OI, Chauhan M, Kim HJ, Jeong WC, Wi H, Oh TI, Woo EJ. Fast conductivity imaging in magnetic resonance electrical impedance tomography (MREIT) for RF ablation monitoring. Int J Hyperth 2014; 30:447-455.

6. Ferree TC, Eriksen KJ, Tucker DM. Regional head tissue conductivity estimation for improved EEG analysis. IEEE Trans Biomed Eng 2000; 47:1584-1592.

7. Akalin Acar Z, Acar CE, Makeig S. Simultaneous head tissue conductivity and EEG source location estimation. Neuroimage 2016;124:168180.

8. Thielscher A, Kammer T. Linking physics with physiology in TMS: a sphere field model to determine the cortical stimulation site in TMS. Neuroimage 2002;17:1117-1130.

9. Mandija S, Petrov PI, Neggers SWF, de Weijer AD, Luijten PR, van den Berg CAT. MR Guidance of TMS for a Patient Specific Treatment Plan: MR Based TMS Field Measurements and Electromagnetic 
Simulations. In Proceedings of the 23rd Annual Meeting of ISMRM, Toronto, Canada, 2015. p. 931.

10. Neggers SFW, Petrov PI, Mandija S, Sommer IEC, van den Berg CAT. Understanding the biophysical effects of transcranial magnetic stimulation on brain tissue. In: Bestmann S, editor. Progress in brain research. Vol. 222. Amsterdam: Elsevier; 2015. pp. 229-259.

11. Surowiec AJ, Stuchly SS, Barr JR, Swarup A. Dielectric properties of breast carcinoma and the surrounding tissues. IEEE Trans Biomed Eng 1988;35:257-263.

12. Joines WT, Zhang Y, Li C, Jirtle RL. The measured electrical properties of normal and malignant human tissues from 50 to $900 \mathrm{MHz}$. Med Phys 1994;21:547-550.

13. Oh TI, Jeong WC, McEwan A, Park HM, Kim HJ, Kwon OI, Woo EJ. Feasibility of magnetic resonance electrical impedance tomography (MREIT) conductivity imaging to evaluate brain abscess lesion: In vivo canine model. J Magn Reson Imaging 2013;38:189-197.

14. Balidemaj E, van Lier ALHMW, Crezee H, Nederveen AJ, Stalpers LJA, van den Berg CAT. Feasibility of electric property tomography of pelvic tumors at 3T. Magn Reson Med 2015;73:1505-1513.

15. Hancu I, Roberts JC, Bulumulla S, Lee S-K. On conductivity, permittivity, apparent diffusion coefficient, and their usefulness as cancer markers at MRI frequencies. Magn Reson Med 2015;73:2025-2029.

16. Shin J, Kim MJ, Lee J, Nam Y, Kim M, Choi N, Kim S, Kim D-H. Initial study on in vivo conductivity mapping of breast cancer using MRI. J Magn Reson Imaging 2015;42:371-378.

17. Kranjc M, Markelc B, Bajd F, Čemažar M, Serša I, Blagus T, Miklavčič D. In situ monitoring of electric field distribution in mouse tumor during electroporation. Radiology 2015;274:115-123.

18. Kim S-Y, Shin J, Kim D-H, Kim MJ, Kim E-K, Moon HJ, Yoon JH. Correlation between conductivity and prognostic factors in invasive breast cancer using magnetic resonance electric properties tomography (MREPT). Eur Radiol 2015:1-10.

19. Voigt T, Katscher U, Doessel O. Quantitative conductivity and permittivity imaging of the human brain using electric properties tomography. Magn Reson Med 2011;66:456-466.

20. van Lier ALHMW, Brunner DO, Pruessmann KP, Klomp DWJ, Luijten PR, Lagendijk JJW, van den Berg CAT. B1+ Phase mapping at $7 \mathrm{~T}$ and its application for in vivo electrical conductivity mapping. Magn Reson Med 2012;67:552-561.

21. Katscher U, Kim D-H, Seo JK. Recent progress and future challenges in MR electric properties tomography. Comput Math Methods Med 2013;2013:1-11.

22. Zhang X, Liu J, He B. Magnetic-resonance-based electrical properties tomography: a review. IEEE Rev Biomed Eng 2014;7:87-96.

23. Hafalir FS, Oran OF, Gurler N, Ider YZ. Convection-reaction equation based magnetic resonance electrical properties tomography (crMREPT). IEEE Trans Med Imaging 2014;33:777-793.

24. Gurler N, Ider YZ. Gradient-based electrical conductivity imaging using MR phase. Magn Reson Med 2017;77:137-150.

25. Ider YZ, Birgul O. Use of the magnetic field generated by the internal distribution of injected currents for electrical impedance tomography (MR-EIT). Elektr Turk J Electr Comput Sci 1998;6:215-225.

26. Woo EJ, Seo JK. Magnetic resonance electrical impedance tomography (MREIT) for high-resolution conductivity imaging. Physiol Meas 2008;29:R1-R26.

27. Kim HJ, Kim YT, Minhas AS, Jeong WC, Woo EJ, Seo JK, Kwon OJ. In vivo high-resolution conductivity imaging of the human leg using MREIT: the first human experiment. IEEE Trans Med Imaging 2009; 28:1681-1687.

28. Oran OF, Ider YZ. Magnetic resonance electrical impedance tomography (MREIT) based on the solution of the convection equation using FEM with stabilization. Phys Med Biol 2012;57:5113-5140.

29. Seo JK, Woo EJ. Electrical tissue property imaging at low frequency using MREIT. IEEE Trans Biomed Eng 2014;61:1390-1399.

30. Ozparlak L, Ider YZ. Induced current magnetic resonance-electrical impedance tomography. Physiol Meas 2005;26:S289-S305.

31. van Lier ALHMW, van den Berg CAT, Katscher U. Measuring Electrical Conductivity at Low Frequency Using the Eddy Currents Induced by the Imaging Gradients. In Proceedings of the 20th Annual Meeting of ISMRM, Melbourne, Victoria, Australia, 2012. p. 3467.

32. Oran OF, Hafalir FS, Gurler N, Ider YZ. Convection-Reaction Equation Based Low-Frequency Conductivity Imaging Using Readout Gradient Induced Eddy Currents. In Proceedings of the 21st Annual Meeting of ISMRM, Salt Lake City, Utah, USA, 2013. p. 4188.
33. Liu C, Li W, Argyridis I. Imaging Electric Conductivity and Conductivity Anisotropy via Eddy Currents Induced by Pulsed Field Gradients. In Proceedings of the 22nd Annual Meeting of ISMRM, Milan, Italy, 2014. p. 638.

34. Mandija S, van Lier ALHMW, Thielscher A, Antunes A, Neggers SFW, Luijten PR, van den Berg CAT. Characterizing Electrical Interactions of Tissue with Time-Varying Gradient Fields: Simulations and Measurements. In Proceedings of the 22nd Annual Meeting of ISMRM, Milan, Italy, 2014. p. 639.

35. Eroglu HH, Eyuboglu BM. Induced Current Magnetic Resonance Electrical Impedance Tomography with z-Gradient Coil. In Engineering in Medicine and Biology Society (EMBC), 36th Annual International Conference of the IEEE, 2014. pp. 1143-1146.

36. Su J, Zheng B, Li SFY, Huang SY. Further Study of the Effects of a Time-Varying Gradient Fields on Phase Maps-Theory and Experiments. In Proceedings of the 23rd Annual Meeting of ISMRM, Toronto, Canada, 2015. p. 3291.

37. Oran OF, Gurler N, Ider YZ. Feasibility of Conductivity Imaging Based on Slice Selection and Readout Gradient Induced Eddy-Currents. In Proceedings of the 23rd Annual Meeting of ISMRM, Toronto, Canada, 2015. p. 930.

38. Mandija S, van Lier ALHMW, Katscher U, Petrov PI, Neggers SFW, Luijten PR, van den Berg CAT. A geometrical shift results in erroneous appearance of low frequency tissue eddy current induced phase maps. Magn Reson Med 2016;76:905-912.

39. Gibbs E, Liu C. Feasibility of imaging tissue electrical conductivity by switching field gradients with MRI. Tomography 2015;1:125-135.

40. King KF. Eddy-current Compensation. In: Bernstein MA, King KF, Zhou XJ, editors. Handbook of MRI Pulse Sequences. Burlington, MA: Elsevier; 2004. pp. 316-331.

41. Jezzard P, Barnett AS, Pierpaoli C. Characterization of and correction for eddy current artifacts in echo planar diffusion imaging. Magn Reson Med 1998;39:801-812.

42. Haselgrove JC, Moore JR. Correction for distortion of echo-planar images used to calculate the apparent diffusion coefficient. Magn Reson Med 1996;36:960-964.

43. Katscher U, Djamshidi K, Voigt T, Ivancevic M, Hiroyuki A, Newstead G, Keupp J. Estimation of Breast Tumor Conductivity Using Parabolic Phase Fitting. In Proceedings of the 20th Annual Meeting of ISMRM, Melbourne, Australia, 2012. p. 3482.

44. Scott GC, Joy MLG, Armstrong RL, Henkelman RM. Measurement of nonuniform current density by magnetic resonance. IEEE Trans Med Imaging 1991;10:362-374.

45. Park C, Lee BI, Kwon OI. Analysis of recoverable current from one component of magnetic flux density in MREIT and MRCDI. Phys Med Biol 2007;52:3001-3013.

46. Wen H. Non-invasive quantitative mapping of conductivity and dielectric distributions using the RF wave propagation effects in high field MRI. In Yaffe MJ, Antonuk LE, editors. In Proceedings of SPIE, Vol. 5030, 2003. pp. 471-477.

47. Gudbjartsson H, Patz S. The rician distribution of noisy MRI data. Magn Reson Med 1995;34:910-914.

48. Lee S-K, Bulumulla S, Hancu I. Theoretical investigation of random noise-limited signal-to-noise ratio in MR-based electrical properties tomography. IEEE Trans Med Imaging 2015;34:2220-2232.

49. Jackson JD. From Lorenz to Coulomb and other explicit gauge transformations. Am J Phys 2002;70:917-928.

50. Haacke EM, Brown RW, Thompson MR, Venkatesan R. Magnetic resonance imaging: Physical principles and sequence design. New York: John Wiley \& Sons; 1999. 843 p.

51. Savitzky A, Golay MJE. Smoothing and differentiation of data by simplified least squares procedures. Anal Chem 1964;36: 1627-1639.

52. John V, Knobloch P. On spurious oscillations at layers diminishing (SOLD) methods for convection-diffusion equations: Part I-a review. Comput Methods Appl Mech Eng 2007;196:2197-2215.

53. Bennett D. NaCl doping and the conductivity of agar phantoms. Mater Sci Eng C 2011;31:494-498.

54. Kato H, Ishida T. Development of an agar phantom adaptable for simulation of various tissues in the range 5-40 MHz. Phys Med Biol 1987;32:221-226

55. Iizuka K. An agar-agar chamber for study of electromagnetic waves in an inhomogeneous medium. IEEE Trans Antennas Propag 1971;19: 365-377. 
56. Dietrich O, Raya JG, Reeder SB, Reiser MF, Schoenberg SO. Measurement of signal-to-noise ratios in MR images: influence of multichannel coils, parallel imaging, and reconstruction filters. J Magn Reson Imaging 2007;26:375-385.

57. Tschumperle D, Deriche R. Diffusion PDEs on vector-valued images. IEEE Signal Process Mag 2002;19:16-25.

58. Horsfield MA. Mapping eddy current induced fields for the correction of diffusion-weighted echo planar images. Magn Reson Imaging 1999;17:1335-1345.

59. Bastin ME, Armitage PA. On the use of water phantom images to calibrate and correct eddy current induced artefacts in MR diffusion tensor imaging. Magn Reson Imaging 2000;18:681-687.
60. Tuch DS, Wedeen VJ, Dale AM, George JS, Belliveau JW. Conductivity tensor mapping of the human brain using diffusion tensor MRI. Proc Natl Acad Sci 2001;98:11697-11701.

61. Seo JK, Pyo HC, Park C, Kwon O, Woo EJ. Image reconstruction of anisotropic conductivity tensor distribution in MREIT: computer simulation study. Phys Med Biol 2004;49:4371-4382.

62. Nam HS, Kwon OI. Axial anisotropic conductivity imaging based on projected current density in MREIT. IEEE Trans Med Imaging 2010;29:781-789.

63. Degirmenci E, Eyuboglu BM. Practical realization of magnetic resonance conductivity tensor imaging (MRCTI). IEEE Trans Med Imaging 2013;32:601-608 